\title{
Percepção dos acadêmicos de Odontologia sobre câncer bucal
}

\author{
Dental students' perception about oral cancer \\ Percepción de los estudiantes de odontología sobre el cáncer oral \\ Jéssica de França VIRGÍ́NIO ${ }^{1}$ \\ Flaviana Laís Pereira dos SANTOS ${ }^{2}$ \\ José Jhenikártery Maia de OLIVEIRA ${ }^{2}$ \\ Micaella Fernandes FARIAS ${ }^{2}$ \\ Bianca Oliveira TÔRRES ${ }^{3}$ \\ Solange Soares da Silva FÉLIX ${ }^{4}$ \\ Graduada em Odontologia pelo Centro Universitário de João Pessoa - UNIPÊ 58053-000 João Pessoa-PB, Brasil \\ ${ }^{2}$ Graduandos do Curso de Odontologia do Centro Universitário de João Pessoa - UNIPÊ 58053-000 João Pessoa-PB, Brasil \\ ${ }^{3}$ Professora Mestre do Curso de Odontologia do Centro Universitário de João Pessoa - UNIPÊ 58053-000 João Pessoa-PB, Brasil \\ ${ }^{4}$ Professora Doutora do Curso de Odontologia do Centro Universitário de João Pessoa - UNIPÊ 58053-000 João Pessoa-PB, Brasil
}

\section{Resumo}

Introdução: O câncer bucal é referido como um dos maiores problemas de saúde pública no mundo, sendo dever do cirurgião-dentista estar preparado para utilizar estratégias de detecção precoce de tal condição, atentando-se aos fatores predisponentes como o tabaco e o álcool. Objetivo: Avaliar a percepção dos acadêmicos dos $9^{\circ}$ e $10^{\circ}$ períodos do curso de Odontologia sobre o câncer bucal. Metodologia: A pesquisa foi desenvolvida nas dependências do referido curso com amostra correspondente a 74 participantes, de ambos os sexos e maiores de idade. Como instrumento de coleta de dados foi utilizado um questionário, contendo 13 questões objetivas relacionadas aos objetivos do estudo. Resultados: A maioria dos participantes afirmou ter conhecimento sobre o problema com informações adquiridas na graduação e grande parte afirma procurar identificar câncer bucal na primeira consulta do paciente. Daqueles que não o fazem, $28,57 \%$ não sabem e $21,43 \%$ consideram desnecessário esse exame, a maioria considera o cirurgião-dentista de alta importância na prevenção e detecção do câncer bucal. Conclusão: Dado o exposto, se faz necessário a orientação aos acadêmicos sobre a importância da participação do cirurgião-dentista na prevenção e diagnóstico precoce do câncer bucal para que este profissional conquiste maior confiança da população com maior qualidade e resolutividade no atendimento, melhorando prognóstico da doença.

Descritores: Neoplasias Bucais; Odontologia; Diagnóstico.

\section{Abstract}

Introduction: Oral cancer is referred to as one of the biggest public health problems in the world, and it is the duty of the dental surgeon to be prepared to use strategies for early detection of this condition, paying attention to predisposing factors such as tobacco and alcohol. Objective: To evaluate the perception of students from the 9th and 10th periods of the Dental Course on oral cancer. Methodology: The research was developed in the dependencies of the referred course with a sample corresponding to 74 participants, both sexes and older. As a data collection instrument, a questionnaire containing 13 objective questions related to the study objectives was used. Results: Most participants said they had knowledge about the problem and this information acquired at graduation, and most participants claim to identify oral cancer at the first consultation of the patient. Of those who do not, $28.57 \%$ do not know and $21.43 \%$ consider this examination unnecessary, most consider the dentist of high importance in the prevention and detection of oral cancer. Conclusion: Given the above, it is necessary to provide guidance to academics about the importance of dentist participation in the prevention and early diagnosis of oral cancer so that this professional gains greater confidence of the population with higher quality and resoluteness in care, giving conditions to reversal of the epidemiological picture of the disease.

Descriptors: Mouth Neoplasms; Dentistry; Diagnosis.

\section{Resumen}

Introducción: El cáncer oral se conoce como uno de los mayores problemas de salud pública en el mundo, y es deber del cirujano dental estar preparado para usar estrategias para la detección temprana de esta afección, prestando atención a factores predisponentes como el tabaco y el alcohol. Objetivo: Evaluar la percepción de los estudiantes de los períodos noveno y décimo del curso dental sobre cáncer oral. Metodología: La investigación se desarrolló en las dependencias del curso referido con una muestra correspondiente a 74 participantes, de ambos sexos y mayores. Como instrumento de recolección de datos, se utilizó un cuestionario que contenía 13 preguntas objetivas relacionadas con los objetivos del estudio. Resultados: La mayoría de los participantes dijeron que tenían conocimiento sobre el problema y esta información adquirida en la graduación, y la mayoría de los participantes afirman identificar el cáncer oral en la primera consulta del paciente. De los que no lo hacen, el $28.57 \%$ no lo sabe y el $21.43 \%$ considera que este examen es innecesario, la mayoría considera al dentista de gran importancia en la prevención y detección del cáncer oral. Conclusión: Teniendo en cuenta lo anterior, es necesario brindar orientación a los académicos sobre la importancia de la participación del dentista en la prevención y el diagnóstico temprano del cáncer oral para que este profesional gane una mayor confianza de la población con mayor calidad y resolución en la atención, dando condiciones a inversión del cuadro epidemiológico de la enfermedad.

Descriptores: Neoplasias de la Boca; Odontologia; Diagnóstico.

\section{INTRODUÇÃO}

Nos últimos tempos, houve um declínio das taxas de mortalidade por doenças infecciosas, em contrapartida, têm aumentado as taxas por doenças não infecciosas, a exemplo das doenças cardíacas, das mortes violentas e das neoplasias. Em relação às neoplasias, no Brasil, o câncer vem ocupando, cada vez mais, um papel de destaque na mortalidade ${ }^{1}$.

$$
\text { Além da questão da mortalidade, a }
$$
morbidade associada a estas neoplasias tem impacto importante na qualidade de vida dos pacientes, devido ao grande número de diagnósticos tardios que reduzem as possibilidades de cura e aumentam as mutilações decorrentes do tratamento ${ }^{1}$.
O câncer bucal é referido como um dos maiores problemas de saúde pública mundial ${ }^{2}$. Considerando os profissionais mais capacitados a promover informações sobre o câncer bucal, o cirurgião-dentista deve estar preparado para utilizar estratégias de detecção precoce do câncer bucal, reduzindo sua incidência e mortalidade ${ }^{3}$.

Contrariamente a outros tipos de câncer que requerem ferramentas mais sofisticadas para o diagnóstico, o câncer bucal é facilmente detectado em um exame clínico de rotina, pois na maioria das vezes é precedido por lesões pré-malignas. A carência de conhecimentos ou a pouca sensibilidade 
de cirurgiões-dentistas em relação ao câncer, é mais preocupante do que o retardo de muitos pacientes em procurar auxílio profissional para o problema.

$\mathrm{O}$ cirurgião-dentista tem papel preponderante na detecção precoce do câncer bucal por ser a boca facilmente acessível ao exame clínico, à biópsia e ao tratamento, o que facilita a detecção, na maioria das vezes ${ }^{4}$.

Ao realizar exames clínicos acurados, incluindo avaliação de linfonodos e orofaringe, a cada retorno, o diagnóstico deverá ser o mais rápido possível e, as lesões suspeitas ao serem confirmadas, receberão o devido tratamento em tempo hábil. Além disso, os profissionais precisam orientar seus pacientes e à população em geral sobre os fatores de risco para o câncer bucal.

Infelizmente, a maioria dos cirurgiõesdentistas não se sentem preparados ou não possuem o devido interesse para orientação sobre prevenção ao câncer bucal, especialmente sobre a adoção de hábitos saudáveis, dentre estes, o não consumo de tabaco e de álcool. Estes fatores de risco são reconhecidos mundialmente como os mais importantes para o desenvolvimento da doença. $\mathrm{O}$ alto índice de mortalidade por câncer bucal é inadmissível quando a prevenção e o diagnóstico precoce podem ser implementados ${ }^{5}$.

A prevenção primária, os diagnósticos clínicos e histopatológicos precoces, além da reabilitação maxilo-facial estão no quadro de obrigações do cirurgião-dentista e, cada profissional deve conhecer o assunto, suas possibilidades de atuação e os limites legais entre as profissões que atuam no tratamento do câncer bucal ${ }^{6}$.

Vários estudos sobre o conhecimento de diferentes populações a respeito do câncer vêm acontecendo no mundo, e os resultados têm apontado um baixo nível de conhecimento ${ }^{7}$. Um levantamento realizado nos EUA em 1990 demonstrou pouco conhecimento sobre fatores desencadeantes do câncer bucal. A partir dos resultados o Center for Disease Control and Prevention decidiu desenvolver estratégias no intuito de reduzir o consumo de cigarros e de bebidas alcoólicas, especialmente aos jovens ${ }^{3}$.

A prevalência da utilização do tabaco e do álcool pelos profissionais da saúde pode prejudicar o controle adequado desses fatores de risco. Levando em consideração os aspectos abordados, surgiu o questionamento quanto ao reconhecimento do tabagismo e o consumo de álcool como fatores de risco para o câncer bucal, pelos acadêmicos do curso de Odontologia regularmente matriculados no último ano do curso. O estudo se justifica pela importância e necessidade de buscar dados sobre o conhecimento e as atitudes dos acadêmicos sobre a temática. Diante desse contexto emerge o questionamento sobre a percepção dos acadêmicos de Odontologia sobre câncer bucal.
O estudo teve por objetivo geral avaliar a percepção dos acadêmicos de Odontologia sobre câncer bucal e por objetivos específicos, conhecer as atitudes dos participantes do estudo perante seus pacientes em relação ao tabagismo e consumo de álcool.

\section{MATERIAL E MÉTODO}

O estudo classifica-se como método indutivo, exploratório, descritivo e bibliográfico. Do ponto de vista de abordagem do problema, a pesquisa desenvolvida apresenta um caráter qualitativo, na qual se utilizou como instrumento de coleta de dados um questionário com 13 perguntas objetivas que compreendiam a percepção dos acadêmicos sobre o câncer bucal, obtendo como amostra 74 acadêmicos, regularmente matriculados no último ano do curso de Odontologia. O conteúdo do questionário abrangeu informações sociodemográficas dos participantes (idade, sexo e período), prevenção, orientação e diagnóstico do câncer, respeitando os aspectos éticos e as orientações preconizadas pela Resolução CNS/MS 466/2012. Os questionários foram aplicados sem controle de tempo para o preenchimento, a fim de não causar desconforto aos participantes. Após obtenção dos dados, os mesmos foram registrados na forma de banco de dados e analisados por meio de estatística descritiva. Os resultados foram apresentados em frequências e percentuais distribuídos em gráficos e tabelas, para a discussão dos mesmos. (CAAE: 19309512.8.0000.5176).

\section{RESULTADOS}

O universo dessa pesquisa compreendeu todos os alunos do curso de Odontologia, de ambos os sexos, maiores de dezoito anos de idade, matriculados no $9^{\circ}$ e no $10^{\circ}$ períodos do curso, o que totalizava 90 alunos. A amostra prevista, inicialmente, correspondia a 74 participantes $(82,22 \%)$. Entretanto, só foi possível incluir 59 participantes no estudo, ou seja, $65,55 \%$ do universo. O restante da amostra prevista (15 acadêmicos) não concordou em participar do estudo, ou entregou o questionário parcialmente preenchido, devido a isso, foram excluídos da amostra. Dos acadêmicos pesquisados, $20(34,00 \%)$ são homens e $39(66,00$ $\%)$ são mulheres. Os participantes do estudo estão distribuídos de acordo com o período em curso, sendo o maior percentual do $10^{\circ}$ período $(52,54 \%)$.

A Tabela 1 demonstra os fatores risco associados ao câncer de boca, assinalado pelos acadêmicos que participaram do estudo. Observa-se que todos os participantes $(n=59)$ consideram o fumo (tabaco) um fator de risco para o câncer bucal. Em seguida, a exposição ao sol $(98,31 \%)$ e o álcool $(91,53 \%)$. Importante considerar ainda, o percentual de respostas em relação aos fatores hereditários $(44,07 \%)$. No que se refere à prevenção do câncer, 
houve um elevado percentual de participantes $(94,92 \%)$ que acreditam que o câncer pode ser prevenido.

Tabela1 - Distribuição absoluta e percentual das respostas dos participantes do estudo quanto à questão "Quais os fatores de risco que você associa ao câncer de boca?”, João Pessoa/PB, 2014

\begin{tabular}{lcc}
\hline $\begin{array}{c}\text { Fatores de risco associados } \\
\text { ao câncer de boca }\end{array}$ & F & \% \\
\hline Fumo & 59 & 100,00 \\
Dieta & 17 & 28,81 \\
Álcool & 54 & 91,53 \\
Sol & 58 & 98,31 \\
Estresse & 09 & 15,25 \\
Dentes estragados & 03 & 5,08 \\
Má higiene & 20 & 33,90 \\
Fatores Hereditários & 26 & 44,07 \\
Ingestão de carne vermelha & 01 & 1,69 \\
\hline Fonte: Pesquisa própria. & \multicolumn{2}{l}{} \\
Obs. O participante poderia assinalar mais de um fator de risco. &
\end{tabular}

Contemplando o objetivo da pesquisa, os participantes foram indagados acerca do conhecimento sobre câncer bucal. Observa-se um percentual bem elevado $(98,31 \%)$ de acadêmicos que afirmam ter conhecimento sobre o assunto em questão (Figura 1). Analisando a distribuição das respostas relacionadas ao conhecimento dos participantes sobre câncer bucal, percebe-se que $50,85 \%$ consideram bom e $38,99 \%$ consideram regular o seu conhecimento acerca do assunto.

Dos acadêmicos entrevistados, $98 \%$ afirmaram ter obtidos informações sobre o câncer bucal durante a graduação, seguindo-se de $20 \%$ daqueles que receberam informação da imprensa. Os que receberam orientação em outro local (5\%) citaram os livros, revistas e artigos científicos. Um levantamento foi realizado em relação ao comportamento dos acadêmicos quanto à exames que visem a identificação do câncer bucal na primeira consulta. Como visto na Figura 2, 76,27\% dos entrevistados afirmaram que buscam sinais de alterações no primeiro contato. Dos participantes que não realizam os exames para identificação do câncer bucal em seus pacientes, $28,57 \%$ não sabem fazê-lo e 21,43\% consideram desnecessário esse exame, 50\% assinalou outro motivo (Figura 3). Considerando o conhecimento dos acadêmicos quanto ao tema, quando questionados sobre o tipo de câncer mais comum, $83,05 \%$ respondeu que o carcinoma espinocelular é o tipo de câncer mais frequente na cavidade bucal e com relação à região anatômica onde o câncer bucal é visto com mais frequência, o maior percentual de respostas foi língua $(59,32 \%)$, seguindo-se do assoalho da boca $(49,15 \%)$ e do palato $(40,68 \%)$.

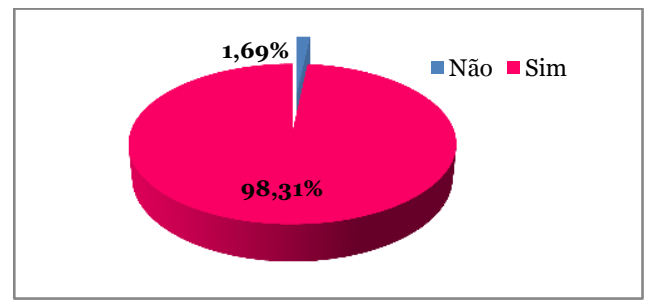

Figura 1 - Distribuição percentual das respostas dos participantes do estudo em relação à questão "Você tem conhecimento sobre câncer de boca?” João Pessoa/PB, 2014. (Fonte: Pesquisa própria)
Dado a opinião dos acadêmicos sobre a importância do cirurgião-dentista em relação à prevenção e detecção precoce do câncer bucal, $89,84 \%$ dos entrevistados consideram o cirurgião dentista fundamental na prevenção e detecção do câncer bucal, como visto na Figura 4.

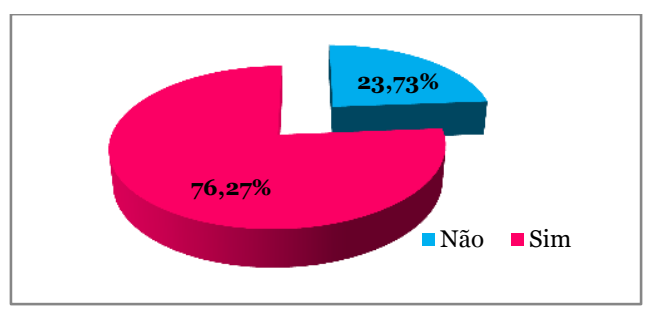

Figura 2 - Distribuição percentual das respostas dos participantes do estudo quanto à questão "Na primeira consulta odontológica dos seus pacientes, você realiza exames procurando identificar câncer bucal?’ João Pessoa/PB, 2014 (Fonte: Pesquisa própria)

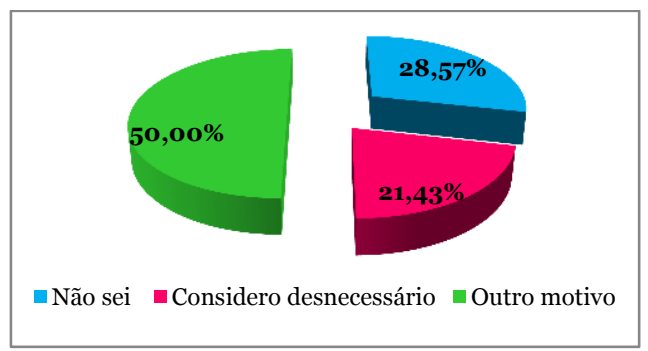

Figura 3 - Distribuição das respostas dos participantes do estudo em relação ao motivo da não realização de exame de câncer bucal em seus pacientes. João Pessoa/PB, 2014 (Fonte: Pesquisa própria)

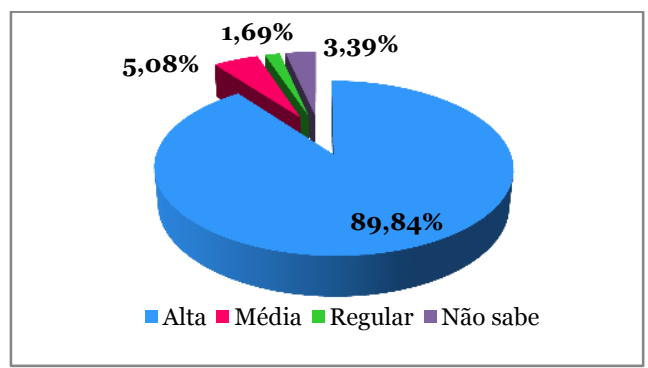

Figura 4 - Distribuição percentual das respostas dos participantes do estudo sobre a importância do cirurgião-dentista na prevenção e detecção precoce do câncer bucal. João Pessoa/PB, 2014 (Fonte: Pesquisa própria)

\section{DISCUSSÃO}

Um aspecto fundamental para realizar o diagnóstico precoce do câncer bucal consiste no conhecimento de suas manifestações iniciais ${ }^{8,9}$. O papel do profissional de saúde bucal é primordial no estabelecimento de programas de prevenção ao câncer bucal, pois é ele que tem melhor acesso aos locais anatômicos onde ocorrem a doença ${ }^{10}$. A prevenção primária, os diagnósticos clínicos e histopatológicos precoces, a reabilitação maxilofacial estão no quadro de obrigações da odontologia e cada cirurgião-dentista deve conhecer o assunto, suas possibilidades de atuação e os limites legais entre as profissões que atuam no tratamento do câncer bucal ${ }^{6}$.

Apesar da quase totalidade dos participantes do estudo afirmarem ter obtido informação sobre o câncer bucal na graduação, as faculdades de Odontologia devem enfatizar mais o conteúdo, despertando a consciência para o problema desde os bancos escolares, incutindo uma filosofia preventiva 
nos alunos, que se refletirá posteriormente na sua prática cotidiana, independente de uma atuação como generalista ou como especialista ${ }^{11,12}$.

$\mathrm{Na}$ literatura, o consumo de álcool e tabaco, história de câncer prévio, predisposição genética, exposição solar são reconhecidos como fatores de riscos ${ }^{10,13}$. Os estudos pesquisados encontraram frequências bem semelhantes em relação aos fatores de risco álcool e tabaco, ${ }^{9,14-20}$. Mas se por um lado os profissionais reconhecem com facilidade os dois principais fatores de risco para o câncer bucal, não se sabe até que ponto estes hábitos são investigados em seus pacientes. Cabe à comunidade odontológica, principalmente no ambiente acadêmico, garantir que todos os pacientes adultos sejam regularmente examinados para o câncer bucal, além de educá-los com relação à real necessidade deste exame e sobre os fatores de risco conhecidos para esse tipo de doença ${ }^{3}$. Sem informações exatas e adequadas, as pessoas não podem realizar ações preventivas, tampouco se pode esperar que elas detenham decisões inteligentes com relação à sua saúde.

$\mathrm{Na}$ literatura científica pesquisada, há um consenso entre os autores em relação ao tipo de câncer mais comum na cavidade bucal, o carcinoma espinocelular ou carcinoma de células escamosas ou ainda, carcinoma epidermóide ${ }^{21-25}$. Interessante ressaltar que apesar de apenas $13,56 \%$ dos participantes responderem que não sabem ou não responderam, é preocupante visto que pode comprometer a detecção precoce da neoplasia.

Outros estudos também consideram que a língua é a região anatômica mais acometida por tal condiçãa ${ }^{15,20}$, este resultado traz uma importante reflexão acerca do exame clínico do paciente, pois na maioria das vezes os cirurgiões-dentistas não examinam a língua de seus pacientes, puxando-a para fora, verificando os bordos e a parte inferior. Desta forma, muitas lesões suspeitas e até mesmo carcinomas já instalados podem passar despercebidos ao olhar do profissional, comprometendo mais uma vez o diagnóstico precoce do câncer bucal.

Apesar de considerarem alta a importância do cirurgião-dentista na prevenção e detecção precoce do câncer bucal, a maioria dos profissionais de saúde bucal expõe limitações quanto à prática de orientar os pacientes sobre os malefícios do cigarro e do álcool ${ }^{19,25}$

\section{CONCLUSÃO}

O conhecimento sobre o câncer de boca mostrou-se inconsistente em alguns pontos, com isso, o estudo mostra a necessidade de pensar em estratégias que promovam melhorias referentes ao conhecimento desses acadêmicos. Recomenda-se maior ênfase na orientação aos acadêmicos sobre a importância da participação do cirurgião-dentista na prevenção e diagnóstico precoce do câncer bucal, visto ser este profissional que atua na cavidade bucal. Os cursos de graduação devem capacitar os estudantes a fim de garantir a confiança da população pelo profissional cirurgião-dentista, proporcionando qualidade e resolutividade no atendimento, dando melhores prognósticos a devida condição.

\section{REFERÊNCIAS}

1. Brasil. Ministério da Saúde. Secretaria de Vigilância em Saúde. Análise dos dados de mortalidade de 2001. Brasília, jan. 2004. Acesso em: 02 de abril de 2019. Disponível em: <http://portal.saude.gov.br/portal/arquivos/pdf/ mortalidade\%202001.pdf >.

2. Moore SR, Johnson NW, Pierce AM, Wilson DF. The epidemiology of mouth cancer: a review of global incidence. Oral Dis. 2000;6(2):65-74.

3. Lima AAS, França BHS, Ignácio AS, Baioni CS. Conhecimento de alunos universitários sobre câncer bucal. Rev Bras Cancerologia. 2005; 51(4):283-88.

4. Little JW. Cancer awareness and dentistry. Gen Dent. 2000;48(4):462-65.

5. Vidal AKL, Silveira RCJ, Soares EA, Cabral AC, Caldas Júnior AF, Souza EHA et al. Prevenção e diagnóstico precoce do Câncer de boca: uma medida simples e eficaz. Odontol clín-cient. 2003;2(2):109-14.

6. Almeida FCS, Cazal C, Brandão TB, Araújo ME, Silva DP, Dias RB. Campanha de popularização do autoexame da boca - Universidade de São Paulo, Brasil. Rev bras patol oral. 2005;4(3):147-55.

7. Adlard JW, Hume MJ. Cancer knowledge of the general public in the United Kingdom: survey in a primary care setting and review of the literature. Clin Oncol (R Coll Radiol). 2003;15(4):174-80.

8. Pinheiro SMS, Cardoso JP, Prado FO. Conhecimentos e Diagnóstico em Câncer Bucal entre Profissionais de Odontologia de Jequié, Bahia. Rev Bras Cancerologia. 2010;56(2):195-205.

9. Morais TMN. Câncer de boca: avaliação do conhecimento dos cirurgiões-dentistas quanto aos fatores de risco e procedimentos diagnósticos [dissertação]. São Paulo: Faculdade de Odontologia. Universidade de São Paulo; 2003.

10. Garbin D. Conhecimento dos cirurgiões-dentistas sobre câncer bucal: um estudo de caso na rede pública de Florianópolis - SC [dissertação]. Florianópolis: Universidade Federal de Santa Catarina; 2007.

11. Horowitz AM, Siriphant P, Sheikh A, Child WL. Perspectives of Maryland dentists on oral cancer J Am Dent Assoc. 2001;132(1):65-72.

12. Dib LL, Souza RS, Tortamano N. Avaliação do conhecimento sobre câncer bucal entre alunos de Odontologia, em diferentes unidades da Universidade Paulista. Rev Inst Ciênc Saúde. 2005;23(4):287-95. 
13. Dib LL. Nível de conhecimento e de atitudes preventivas entre universitários do curso de odontologia em relação ao câncer bucal: desenvolvimento de um instrumento de avaliação. Acta Oncol Bras.2004;24(2):628-43.

14. Cannick GF, Horowitz AM, Drury TF, Reed SG, Day TA. Assessing oral cancer knowledge among dental students in South Carolina. J Am Dent Assoc. 2005;136(3):373-78.

15. Clovis JB, Horowitz AM, Poel DH. Oral and pharyngeal cancer: knowledge and opinions of dentists in British Columbia and Nova Scotia. J Can Dent Assoc. 2002;68(7):415-20.

16. Greenwood M, Lowry RJ. Primary care clinicians' knowledge of oral câncer: a study of dentists and doctors in the north east of England. Br Dent J. 2001;191(9):510-12.

17. Patton EE, Zon LI. Taking human cancer genes to the fish: a transgenic model of melanoma in Zebrafish. Zebrafish. 2005;1(4):363-68.

18. Syme SE, Drury TF, Horowitz AM. Maryland dental hygienists' knowledge and opinions of oral cancer risk factors and diagnostic procedures. Oral Dis. 2001;7(3):177-84.

19. Vasconcelos EM. Comportamento dos cirurgiões dentistas das unidades básicas de saúde do município de São Paulo quanto à prevenção e ao diagnóstico precoce do câncer bucal [dissertação]. São Paulo: Faculdade de Odontologia. Universidade de São Paulo; 2006.

20. Yellowitz JA, Horowitz AM, Drury TF, Goodman HS. Survey of U.K. dentists' knowledge and opinions about oral pharyngel cancer. J Am Dent Assoc. 2000;131(5):653-61.

21. Ajayi OF, Adeyemo WL, Ladeinde AL, Ogunlewe MO, Effiom OA, Omitola OG et al. Primary malignant neoplasms of orofacial origin: a retrospective review of 256 cases in a Nigerian tertiary hospital. Int J Oral Maxillofac Surg. 2007; 36(5):403-8.

22. Dedivitis RA, França CM, Mafra ACB, Guimarães FT, Guimarães AV. Características clínico-epidemiológicas no carcinoma espinocelular de boca e orofaringe. Rev Bras Otorrinolaringol. 2004;70(1):35-40.

23. Shiboski CH, Schmidt BL, Jordan RC. Racial disparity in stage at diagnosis and survival among adults with oral cancer in the US. Community Dent Oral Epidemiol. 2007;35(3):233-40.

24. Sawair FA, Al-Mutwakel A, Al-Eryani K, AlSurhy A, Maruyama S, Cheng J et al. High relative frequency of oral squamous cell carcinoma in Yemen: qat and tobacco chewing as its aetiological background. Int $\mathbf{J}$ Environ Health Res. 2007;17(3):185-95.
25. Shiboski CH, Schmidt BL, Jordan RC. Racial disparity in stage at diagnosis and survival among adults with oral cancer in the US. Community Dent Oral Epidemiol. 2007;35(3):233-40.

26. Martins MAT, Marques FGOA, Pavesi VCS, Romão MMA, Lascala CA, Martins MD. Avaliação do conhecimento sobre o câncer bucal entre universitários. Rev Bras Cir Cabeça e Pescoço. 2008;4(37):191-97.

\section{CONFLITO DE INTERESSES}

Os autores declaram não haver conflitos de interesse.

\section{AUTOR PARA CORRESPONDENCIA}

Micaella Fernandes Farias

micaellaff@gmail.com

Submetido em 31/08/2019

Aceito em 08/01/2020 